\title{
High Temperature Operated ( 250 K) Photovoltaic-Photoconductive (PV-PC) Mixed-mode InAs/GaAs Quantum Dot Infrared Photodetector
}

\author{
Shiang-Feng Tang, Shih-Yen Lin, Si-Chen Lee, and Chieh Hsiung Kuan \\ Department of Electrical Engineering, National Taiwan University, Taipei, Taiwan, R. O. C.. \\ Ya-Tung Cherng
}

Materials \& Electro-Optics Division, Chung Shang Institute of Science and Technology, Lung Tan, Taiwan, R. O. C..

\begin{abstract}
The 10 stacked self-assembled InAs/GaAs quantum dot infrared photodetector (QDIP) operated in 2.5 to $7 \mu \mathrm{m}$ range by photovoltaic (PV) and photoconductive (PC) mixed-mode near room temperature $(\sim 250 \mathrm{~K})$ was demonstrated. The specific peak detectivity $\mathrm{D}^{*}$ is $2.4 \times 10^{8} \mathrm{~cm}-\mathrm{Hz}^{1 / 2} / \mathrm{W}$ at 250 $\mathrm{K}$. The confining $\mathrm{Al}_{\mathrm{X}} \mathrm{Ga}_{1-\mathrm{X}} \mathrm{As}$ barrier layers on both sides of stacked QD structure are key to the high temperature operation.
\end{abstract}

\section{Introduction}

In the past several years, $\mathrm{AlGaAs} / \mathrm{GaAs}$ quantum well infrared photodetector (QWIP) had been widely investigated due to its commercial and military applications [1-2]. A similar Structure, InAs/GaAs quantum dot infrared photodetector (QDIP) was proposed and investigated in several reports both in theory and in practical devices [3-7]. The most dramatic effect of using QDIP instead of QWIP to detect mid- and far- infrared signal is its high temperature performance [7]. For QWIP, devices can rarely be operated beyond $100 \mathrm{~K}$. However, QDIP operated over $100 \mathrm{~K}$ had been presented in several reports [6-7]. In this letter, the characteristics of self-assembled InAs QDIP with high temperature operation is presented.

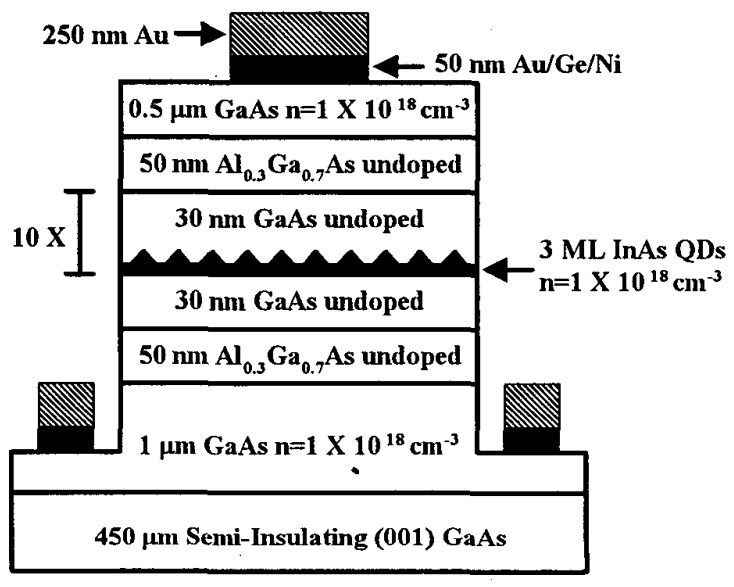

Fig. 1 Schematic diagram showing the device structure of InAs/GaAs QDIP.

\section{Experiments and Discussion}

The self-assembled InAs quantum dots discussed in this letter were grown on (100) GaAs semi-insulating substrate in Stranski-Krastanow (S-K) growth mode using solid-source MBE under As deficient condition. The substrate temperature was kept at $510{ }^{\circ} \mathrm{C}$ during $\mathrm{MBE}$ growth. The detector structure consists of 10 stacks of $3 \mathrm{ML}$ InAs quantum dot layers seperated by $30 \mathrm{~nm}$ GaAs barrier. The self assembled InAs dot was uniformly doped to $1 \times 10^{18} \mathrm{~cm}^{-3}$ with undoped GaAs barrier. The dot density is approximately $4 \times 10^{10} \mathrm{~cm}^{-2}$. Two extra $\mathrm{Al}_{0.3} \mathrm{Ga}_{0.7} \mathrm{As}$ confining layers were grown on both sides of the 10-stacked QD structure to reduce dark current and preserve photoexcited carriers. $0.5 \mu \mathrm{m}$ and $1 \mu \mathrm{m}$ GaAs with doping density $1 \times 10^{18} \mathrm{~cm}^{-3}$ were grown to provide top

\subsection{1}




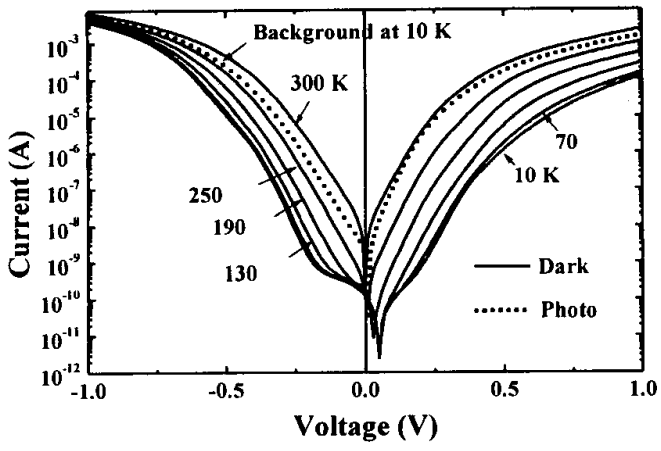

Fig. 2 Dark I-V characteristics of the QDIP from 10 to $300 \mathrm{~K}$. The dotted curve represents the photocurrent measured at $10 \mathrm{~K}$ when the QDIP is illuminated from the ambient background radiation through the cryostat window at $10 \mathrm{~K}$.

and bottom contacts, the schematic device structure is shown in Fig. 1.

Using standard procedure, contact metal evaporation, photolithography and wet etching, $100 \times 100 \mu \mathrm{m}^{2}$ devices are fabricated. The current-voltage (I-V) characteristics of the device were measured by HP4156b semiconductor parameter analyzer. And the spectral response was measured under edge-coupling scheme with APD cryogenics and a Perkin-Elmer S2000 FTIR spectroscope, which is a standard configuration for QWIP measurement [8]. To obtain the absolute value of responsivity, calibration was made by using a black body source and a chopper to chop the infrared signal such that the corresponding photocurrent can be detected by SR830 lock-in amplifier.

Figure 2 displays the dark I-V characteristics of the device. The photocurrent measured at $10 \mathrm{~K}$ while QDIP was illuminated from the ambient background radiation is also shown as dotted curve which appears to be larger than the dark current at $250 \mathrm{~K}$ under the bias of $\pm 1 \mathrm{~V}$ indicating the background-limited-performance temperature (BLIP) to be $250 \mathrm{~K}$. Figs. 3 (a) and (b) show the temperature-dependent photoresponsivity and peak specific detectivity of QDIP at zero bias which gives a direct evidence that this device can be operated at $250 \mathrm{~K}$ in photovoltaic (PV) mode. And from Fig. 3 (b), it is shown that the peak specific detectivity is $2.4 \times$

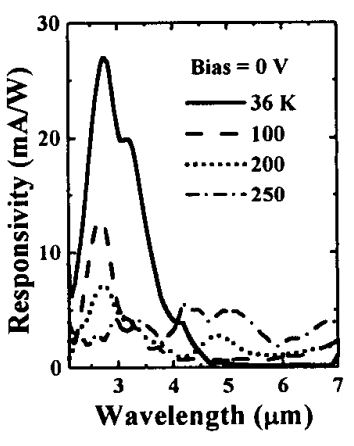

(a)

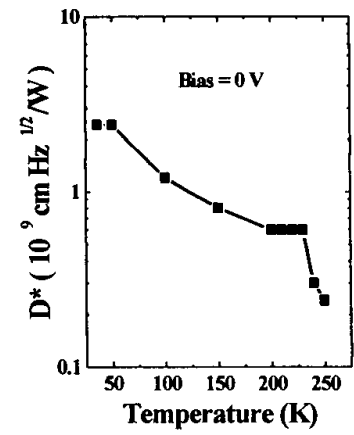

(b)
Fig. 3 (a) The responsivity of QDIP as a function of temperature at zero bias. (b) The specific detectivity of QDIP as a function of temperature at zero bias.

$10^{8} \mathrm{~cm}-\mathrm{Hz}^{1 / 2} / \mathrm{W}$ at $250 \mathrm{~K}$. To our knowledge, this is the highest value for QDIP operated at a temperature beyond 200 $\mathrm{K}$ in the 2.5 to $7 \mu \mathrm{m}$ wavelength range.

Figures 4 (a) and (b) show the responsivity of QDIP operated at $36 \mathrm{~K}$, the peak responsivity of $25 \mathrm{~mA} / \mathrm{W}$ at the range of $2.5 \sim 4 \mu \mathrm{m}$ was observed at zero bias which decreased to almost zero at positive $0.1 \mathrm{~V}$ but increased at negative biases. That is clearly a PV mode operation. However, in the wavelength range from 3.5 to $7 \mu \mathrm{m}$, the peak responsivity is dependent on the biases which vanishes at zero bias, this is a photoconducitivity (PC) mode operation. The peak responsivity at $+0.42 \mathrm{~V}$ is $155 \mathrm{~mA} / \mathrm{W}$ at a wavelength of $5 \mu \mathrm{m}$. The asymmetric photoresponse is attributed to the segregation of doped Si atoms toward the top surface of the QD and results in asymmetric electric field

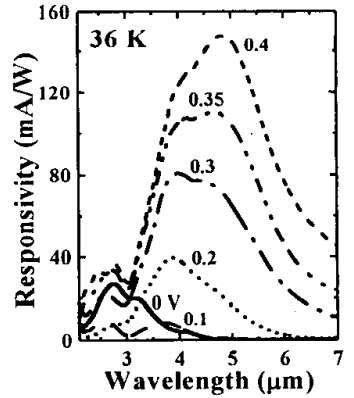

(a)

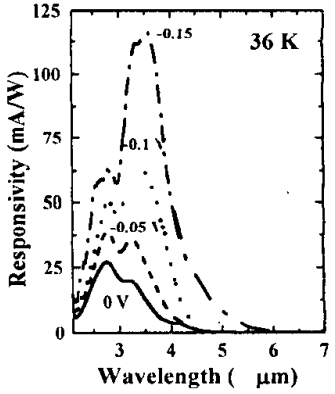

(b)
Fig. 4 The responsivity of QDIP at (a) positive bias from 0 to $0.4 \mathrm{~V}$, (b) negative bias from 0 to $-0.15 \mathrm{~V}$. The temperature is $36 \mathrm{~K}$. 


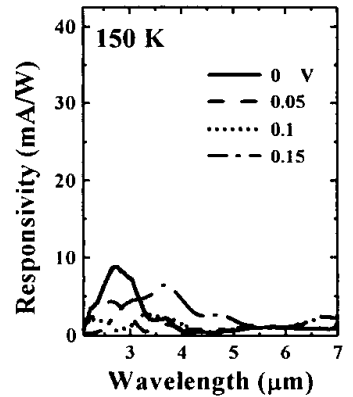

Fig. 5 The responsivity of QDIP at (a) positive bias from 0 to $0.15 \mathrm{~V}$, (b) negative bias from 0 to $-0.15 \mathrm{~V}$. The temperature is $150 \mathrm{~K}$

distribution which lead to charge separation. Figs. 5 (a) and

(b) display the photoresponsivity of QDIP at $150 \mathrm{~K}$, which is dominated by PV mode. The PC mode almost disappears due to the large dark current with increasing bias. The PC mode and PV mode response of the device are attributed to two different mechanisms. The $3.5 \sim 7 \mu \mathrm{m}$ response (PC mode) are due to ground state $E_{0}$ to bound excited state $E_{1}$ transition in the quantum dots as shown in Fig. 6. The photoexcited electrons would tunnel through the sharper barrier formed due to the segregation of Si dopant along the growth direction at positive bias. The $2.5 \sim 4 \mu \mathrm{m}$ responses are due to electron bound state $E_{0}$ to continuum states $E_{2}$ transition, once electrons jump out of the volcanic shaped potential, they are driven by the internal asymmetric electrical field in GaAs toward the substrate side and form a positive open circuit

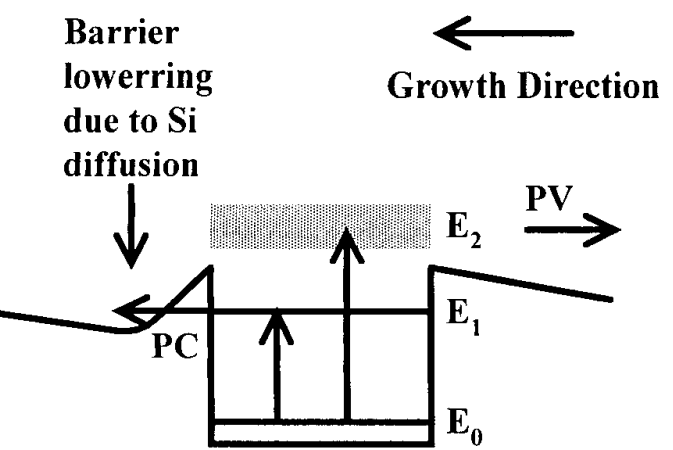

\section{GaAs \\ InAs \\ GaAs}

Fig. 6 The schematic band diagram of the InAs QD surrounded by in GaAs barrier along growth direction, where $E_{0}, E_{1}$ represent the first and second confined ststes and $E_{2}$ is the quasi continuum state. voltage. Thus the PV mode response is formed.

A simple model is proposed to explain the mechanism resulting in high-temperature operation by QDIP. By measuring the recovery of resist after the device is shielded from the glowbar, the recovery time constant is found to be extremely long, i.e., $6.64 \mathrm{sec}$, which suggests that the photoexcited electrons are difficult to be recaptured by QDs unless under rather high temperature. This is the high-photoelectric-gain nature of QDIP. [3] And the $\mathrm{Al}_{0.3} \mathrm{Ga}_{0.7} \mathrm{As}$ blocking layers prevent the photoelectrons to escape from the QD structure and thus photoelectrons are accumulated inbetween. Hence, the electron quasi Fermi level is raised very high as compared to that under dark environment. The photocurrent is much enhanced which explain the high temperature operation of the device.

\section{Conclusion}

In conclusion, with the combination of QD and two blocking barrier, the operation temperature of our QDIP is pushed up to $250 \mathrm{~K}$. With $\mathrm{PC}$ and $\mathrm{PV}$ mode response coexistence, the peak specific detectivity $D^{*}$ is $2.4 \times 10^{8}$ $\mathrm{cm}-\mathrm{Hz}^{1 / 2} / \mathrm{W}$ at $250 \mathrm{~K}$, which is, to our knowledge, the highest value for QDIP operated at a temperature beyond $200 \mathrm{~K}$ in the 2.5 to $7 \mu \mathrm{m}$ range.

\section{References}

(1) S. D. Gunapala, S. V. Bandara, J. K. Liu, W. Hong, M. Sundaram, P. D Maker, R. E. Muller, C. A. Shott, and R. Carralejo, IEEE Trans Electron Devices 45, 1890 (1998)

(2) C. J. Chen, K. K. Choi, W. H. Chang, and D. C. Tsui, Appl. Phys. Lett 72, 7 (1998)

(3) V. Ryzhii, Semicond. Sci. Technol. 11, 759 (1996)

(4) S. Xu, S. J. Chua, T. Mei, X. C. Wang, X. H. Zhang, G. Karunasiri, W. J. Fan, C. H. Wang, J. Jiang, S. Wang, and X. G. Xie, Appl. Phys. Lett. 73, $3153(1998)$ 
(5) D. Pan and E. Towe, Appl. Phys. Lett. 75, 2719 (1999).

(6) S. Kim, H. Mohseni, M. Erdtmann, E. Michel, C. Jelen, and M. Razeghi, Appl. Phys. Lett. 73, 963 (1998).

(7) J. W. Kim, J. E. Oh, S. C. Hong, C. H. Park, and T. K. Yoo, IEEE Trans. Electron Devices Letters 21, 329 (2000).

(8) K. K. Choi, " The Physics of Quantum Well Infrared Photodetectors ", World Scientific Publishing, Singapore, Chap. 6 (1997). 